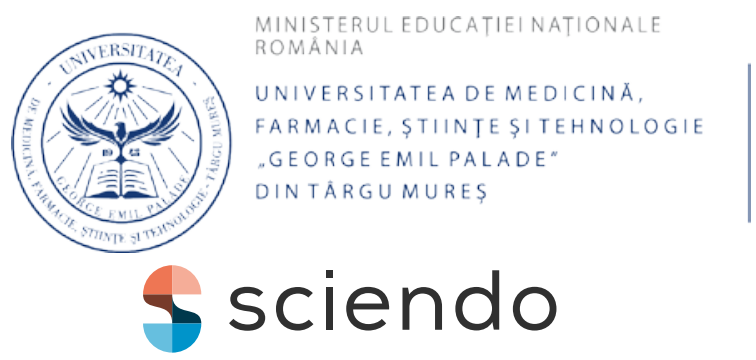

\author{
Acta Marisiensis. Seria Technologica \\ Vol. 18 (XXXIV) no. 2, 2020 \\ ISSN 2668-4217, ISSN-L 2668-4217
}

10.2478/amset-2020-0016

\title{
A SURVEY ON DEVICES EXPLOITING LORA COMMUNICATION
}

\author{
Lorenzo CAROSSO $^{1}$, Luca MATTIAUDA ${ }^{2}$, Marco ALLEGRETTI ${ }^{3}$ \\ ${ }^{1,2,3}$ Polytechnic University of Turin \\ Department of Electronics and Telecommunications \\ Corso Duca degli Abruzzi, 24 - 10129 Torino, ITALY \\ ${ }^{1}$ lorenzo.carosso@polito.it \\ ${ }^{2}$ luca.mattiauda@studenti.polito.it \\ ${ }^{3}$ marco.allegretti@polito.it
}

\begin{abstract}
Information and Communication Technologies (ICT) have experienced a large application in many fields, such as smart homes, health monitoring, environmental monitoring, and a great number of studies is present in literature. In particular, it is expected that the Internet of Things (IoT) will become increasingly pervasive in everyday life. Among different technologies, devices based on Long Range (LoRa) and LoRaWAN stand out due to their relative low cost, low power consumption and large cover range. In this survey, recent papers investigating applications of LoRa modules have been selected. The different usecases are presented with a comparison between communication parameters and results obtained.
\end{abstract}

Key words: LoRa, Network, Communication, Device, Monitoring

\section{Introduction}

By 2024, at least 20 billion connected devices belonging to the Internet of Things (IoT) family are expected to be connected [1]. The market related to IoT and its applications has an estimated growth of 3,900 $-11,000$ trillion dollars by 2025 [2]. The applications of IoT spread in various fields:

(i) Monitoring Greenhouse gases emissions could lead to an overall reduction of $12 \%$ by 2030 [3];

(ii) IoT could help those who cannot physically go into hospitals [4], [5], [6];

(iii) Remotely prevent forests' fire [7], [8], [9];

(iv) Control and automate some process such as public light management [10], and many others.
Given the previous application examples, the area covered by communication systems is a relevant topic. With this this in mind, the technologies that are used are classified as Low Power Wide Area Network (LPWAN). Under this distinction, it is possible to find WI-FI, Bluetooth and Zigbee which have short-range communication, and GSM, WCDMA, NB-IoT, Sigfox and LoRa which have medium or long-range, but only Sigfox and LoRa exploit the Industrial, Scientific and Medical bands, which are unlicensed. Apart from this, LPWAN technologies must satisfy other important requirements, such as simplicity of network's installation, low power consumption and low cost.

$43 \%$ of the overall LPWAN technologies will consist of devices exploiting LoRa communication

(C) 2020 Published by University Press. This is an open access article under the CC BY-NC-ND license (http://creativecommons.org/licenses/CC-BY-4.0/) 
[11]. The structure of this paper is as follows: Section II presents a LoRa and LoRaWAN overview; Section III presents a literature review on the most recent papers about testing devices supporting LoRa; Section IV presents a comparative table and Section $\mathrm{V}$ provides the conclusions.

\section{LoRa and LoRaWAN overview}

LoRa physical layer is a technology patented by Semtech. The LoRa modulation derives from the Chirp Spread Spectrum (CSS) modulation and each symbol is modulated with a chirp signal which frequency sweep coincides with the bandwidth. Then, choosing an appropriate Spreading Factor (SF) it is possible to encode $2 \mathrm{SF}-1$ symbols [12]. As for performance's errors, it has been shown that LoRa modulation gives better results than an FSK one.

The LoRa Alliance has defined the LoRaWAN protocol. It outlines the packet's format (which consists in a preamble, a header, a Cyclic Redundancy Check (CRC), the payload and a payload CRC, the network's operative mode and the encryption. There are three classes in which a network can work, as:

(i) Class A: battery life-time is optimized, uplink is event driven and down- link is limited;

(ii) Class B: similar to Class A but there is more time for downlink;

(iii) Class C: downlink is no more event driven and receiving windows are open continuously.

To assure security, the payload is encrypted using AES encryption with a 128 bits key described in [13]. The key used is specific for each end-device and assigned during fabrication.

\section{Literature review}

In this section, devices supporting the LoRa communication that have been tested in the most recent papers will be presented. Moreover, each usecase will be briefly presented. The module from Semtech and Microchip that supports LoRa communication are the most used within applications presented in literature.

\subsection{SX1272}

In [14] a deep analysis on LoRa communication performances in buildings and in a car park has been conducted, in order to determine the most accurate method to evaluate the path loss in such environments both in condition of line of sight and with obstacles. In this application Multitech mDot supporting the Semtech SX1272 module has been used. Authors implemented a $915 \mathrm{MHz}$ communication with a bandwidth of $500 \mathrm{kHz}$, SF equal to 7 and transmitting power of $10 \mathrm{dBm}$. They estimated a received signal strength indication (RSSI) of $-120 \mathrm{dBm}$ when there are seven floors within transmitter (TX) and receiver (RX), while the sensitivity of SX1272 is $-137 \mathrm{dBm}$. In [15] authors evaluated communication between one TX and a RX in an urban environment to track many environmental indicators such as $\mathrm{CO}_{2}$, temperature, humidity and others. The transceivers consist of two Adafruit Feather 32u4 supporting the LoRa SX1272 chip. They used an output power of $20 \mathrm{dBm}$ at a frequency of $868 \mathrm{MHz}$. In an urban context they reached $1.1 \mathrm{~km}$ with a signal strength of $-95 \mathrm{dBm}$ while in a seven-floors building the least value of RSSI was of $-100 \mathrm{dBm}$ whilst the reduction was not linear; in fact, the floor with the worst RSSI was not the farthest one.

\subsection{SX1276/SX1278}

In [16] a Wireless Sensor Network (WSN) that manages the water irrigation of a garden placed in a city has been developed. Authors claim that up to $30 \%$ of water has been saved with this system. The presented network could support 250 nodes. The core of the nodes is the RFM95W Adafruit supporting the SX1276 LoRa chip and an 868MHz dipole antenna has been used. The scenario has been divided into eight $350 \mathrm{~m}^{2}$ zones and in four zones three nodes have been installed. A single gateway is used to collect data regarding humidity and forward them via $\mathrm{Wi}-\mathrm{Fi}$ to the application's server. Supplying the nodes with 2AA batteries and sending data every 4 hours, the nodes had an autonomy of two months. In [17] a study over the performances of a WSN in an urban environment exploiting the Dragino LoRa HAT and a Raspberry PI, as end nodes for air quality monitoring has been conducted. Authors have tested that SF 10 was the best choice for their use-case. As confirmation of that, also when the adaptive data rate has been enabled, the SF 10 has been automatically set. Six gateways and thirteen transmitting nodes have been employed. A $72.4 \%$ of packet's delivery has been achieved successfully. In [18] a path loss model for the LoRa communication in an urban area through experimental analysis has been evaluated. The system under test has been developed for reporting power consumption to a control center. Operating frequency band was the 920 $\mathrm{MHz}-925 \mathrm{MHz}$ range and end-nodes consist of the STM32 LoRa Discovery Kit (SX1276 chip). The chosen communication's parameters are: bandwidth of $125 \mathrm{MHz}$; adaptive data rate, which means SF could change from 7 to 12 ; transmitting power of $12 \mathrm{dBm}$. The covered area was less than $1 \mathrm{~km}^{2}$.

\subsection{SX1261/SX1262}

In [19], the LoRa communication's performances in Earth-space communication aimed at providing global coverage for IoT devices, also in rural and remote areas have been tested. This type of communication is done exploiting Low Earth Orbit (LEO) satellites as gateways. In these conditions, the free space path loss model can be used and further distances could be reached since transmitter and receiver are always in line of sight during communication. Authors used nodes supporting the Semtech SX1261/SX1262 chip. TX power was of 22 $\mathrm{dBm}$ for an operating of $915 \mathrm{MHz}$ and $14 \mathrm{dBm}$ for the 
$868 \mathrm{MHz}$ frequency. The bandwidth has been set to $125 \mathrm{kHz}$. They tested both monopole and directive antennas, in order to find the SF for which the communication was possible for that kind of application. In [20], an embedded miniaturized system supporting LoRa communication exploiting the SX1261 chip has been developed. Since the system has to be as small as possible and the only supply is a battery charged with a solar panel, the decision of the communication protocol has been done taking into account the best in terms of power consumption. In [21] a sensing node supporting the SX1261 module has been tested. Maximum transmitting power has been used $(14 \mathrm{dBm})$ with SF equal to 7 and a bandwidth of $250 \mathrm{kHz}$. The power consumption was the key feature of this study and authors experienced that a 4.5 years lifetime with a 2,000 mAh could be possible while the node sends GPS data every ten minutes.

Table 1: Comparison between some parameters of the most popular Semtech modules. Data have been taken from [22], [23], [24].

\begin{tabular}{l|lll} 
& SX1272 & SX1276 & SX1261 \\
\hline $\begin{array}{l}\text { Link } \\
\text { Budget }\end{array}$ & $157 \mathrm{~dB}$ & $169 \mathrm{~dB}$ & $170 \mathrm{~dB}$ \\
RX current & $10 \mathrm{~mA}$ & $9.9 \mathrm{~mA}$ & $4.6 \mathrm{~mA}$ \\
& & & \\
Sensitivity & $-137 \mathrm{dBm}$ & $-148 \mathrm{dBm}$ & $-148 \mathrm{dBm}$
\end{tabular}

\subsection{RN2903/RN2483}

The RN2903 and the RN2483 have been released by Microchip. The first is for the $915 \mathrm{MHz}$ frequency, while the second for the $868 \mathrm{MHz}$ frequency. In [25] a network using the RN2903 module as end node for a smart house system has been designed. Sensors and actuators have been used to manage temperature and lights. The test has been done with a fixed gateway and a sensor node has been done move along a campus site. Signal's strength ranged between $-123 \mathrm{dBm}$ and -68 $\mathrm{dBm}$. Authors of [26] tested the LoRa communication's range for the RN2903 module in an urban area with tall buildings. They considered as signal-covered area a zone for which more than $30 \%$ of all sent messages have been correctly received. The maximum range was $250 \mathrm{~m}$ from the gateway using SF $=7$, a bandwidth of $125 \mathrm{kHz}$ and $\mathrm{TX}$ power equal to 14 $\mathrm{dBm}$. In [27] a wearable node with the RN2483 module has been designed. Then, authors tested which SF gave them the best results in terms of distance, with bandwidth equal to $125 \mathrm{kHz}$. After this study, they proofed that it was possible to reach $1.5 \mathrm{~km}$ with $\mathrm{SF}=7$ in [28].

\section{Comparison}

Although the most chosen modules are the ones from Semtech, in all selected papers the presented LoRa modules achieved satisfying requirements for each use-case, both in indoor and outdoor environments, making it a valid choice for IoT applications. Albeit from Tab. 2 it would seem that modules from Microchip [25], [26], [27] have better performances than the Semtech ones, it is important to underline that papers [14], [16], [17], [18], [19], [20], [21] did not investigate maximum reach, but they only focused on feasibility of LoRa communication in specific applications. Due to the attenuation, devices working in the $915 \mathrm{MHz}$ frequency range needed more power than same devices working in the $868 \mathrm{MHz}$ frequency range, in order to obtain similar performances.

Table 2: Comparison between LoRa modules in selected papers (part 1).

\begin{tabular}{|c|c|c|c|}
\hline Paper & use-case & $\begin{array}{l}\text { Bande } \\
(\mathrm{kHz})\end{array}$ & $\mathrm{SF}$ \\
\hline [14] & $\begin{array}{c}\text { multifloor } \\
\text { communication }\end{array}$ & 500 & 7 \\
\hline [15] & urban static WSN & - & - \\
\hline [16] & urban static WSN & - & - \\
\hline [17] & urban static WSN & - & 10 \\
\hline [18] & urban static WSN & 125 & $7 \div 12$ \\
\hline [19] & $\begin{array}{c}\text { Earth-space } \\
\text { communication } \\
\text { (monopole antenna) }\end{array}$ & 125 & 12 \\
\hline [19] & $\begin{array}{c}\text { Earth-space } \\
\text { communication } \\
\text { (directive antenna) }\end{array}$ & 125 & $7 \div 12$ \\
\hline [20] & urban WSN & 250 & 7 \\
\hline [21] & embedded node & 250 & 7 \\
\hline [25] & smart house & - & - \\
\hline [26] & urban WSN & - & 7 \\
\hline [27] & wearable sensor node & 125 & 7 \\
\hline
\end{tabular}

Table 2: Comparison between LoRa modules in selected papers (part 2).

\begin{tabular}{r|cccc} 
Paper & $\begin{array}{c}\text { Frequency } \\
\text { Allocation } \\
(\mathrm{MHz})\end{array}$ & $\begin{array}{c}\mathrm{TX} \\
\text { power } \\
(\mathrm{dBm})\end{array}$ & Range & Module \\
\hline$[14]$ & 915 & 10 & $\begin{array}{r}7 \text { floors } \\
(17.5 \mathrm{~m})\end{array}$ & $\mathrm{SX} 1272$ \\
{$[15]$} & 868 & - & $1.1 \mathrm{~km}$ & $\mathrm{SX} 1272$ \\
{$[16]$} & 868 & - & $350 \mathrm{~m}^{2}$ & $\mathrm{SX} 1276$ \\
{$[17]$} & 868 & - & $1 \mathrm{~km}^{2}$ & $\mathrm{SX} 1276$ \\
{$[18]$} & $923.2-$ & 12 & $40 \mathrm{~m}$ & $\mathrm{SX} 1276$ \\
{$[19]$} & 924.6 & & - & $\mathrm{SX1262}$
\end{tabular}




\begin{tabular}{l|cccc}
{$[19]$} & 915 & 22 & - & SX1262 \\
{$[20]$} & 868 & - & - & SX1261 \\
{$[21]$} & 868 & 14 & - & SX1261 \\
{$[25]$} & 915 & 18.5 & - & RN2903 \\
{$[26]$} & 915 & 12 & $1.5 \mathrm{~km}$ & RN2903 \\
{$[27]$} & 868 & 10 & $1.5 \mathrm{~km}$ & RN2483
\end{tabular}

\section{Conclusion}

This work is intended to investigate the recent trends in device's choice within applications that exploit LoRa modules.

SX1272 has a great strength of the signal in indoor and outdoor urban environments; performance remains good even with tall buildings, but the reduction of the signal is not linear. SX1276 has good performances in rural environments, for example in agriculture systems. SX1278 packet's delivery in urban areas is good.

SX1261 and SX1262 have great performances in Earth-space communications with a lifetime over 4 years.

RN2903 is really good for smart-house systems, but the performance in tall buildings is lower, sine only $30 \%$ of all sent messages is correctly received. Using RN2483 is possible to reach $1.5 \mathrm{~km}$ with SF7.

Further researches could be carried out on maximum distances that can be reached with the Semtech SX1261/2 and there is a lack in the literature of application exploiting the very latest module, such as the Semtech LR1110, that has been released at the beginning of 2020 .

\section{References}

[1] Annual reports archive - Ericsson.

Available:

https://www.ericsson.com/en/investors/financialreports/annual-reports

[2] Manyika, J. (2015), The Internet of Things: Mapping the value beyond the hype, McKinsey Global Institute.

[3] Malmodin, J., \& Bergmark, P. (2015), Exploring the effect of ICT solutions on GHG emissions in 2030, In EnviroInfo and ICT for Sustainability 2015, Atlantis Press.

[4] de Oliveira, L. R., de Moraes, P., Neto, L. P., \& da Conceição, A. F. (2020), Review of LoRaWAN Applications, arXiv preprint arXiv:2004.05871.

[5] Della Mea, V., et al. (2020), A Communication Infrastructure for the Health and Social Care Internet of Things: Proof-of-Concept Study, JMIR Medical Informatics, 8(2), e14583.
[6] Albahri, A. S., et al. (2020), IoT-based telemedicine for disease prevention and health promotion: State-of-the-Art, Journal of Network and Computer Applications, 102873.

[7] Gaitan, N. C., \& Hojbota, P. (2020), Forest Fire Detection System using LoRa Technology, Forest, 11(5).

[8] Sendra, S., et al. (2020), LoRaWAN Network for Fire Monitoring in Rural Environments, Electronics, 9(3), 531.

[9] Gao, D., Xin, J., \& Zhang, F. (2020), A decision tree algorithm for forest fire prediction based on wireless sensor networks, International Journal of Embedded Systems, 13(4), 422-430.

[10] Sánchez Sutil, F., \& Cano-Ortega, A. (2020), Smart public lighting control and measurement system using LoRa network, Electronics, 9(1), 124.

[11] Why LoRa? / Semtech LoRa Technology / Semtech.

Available: https://www. semtech.com/lora/why-lora

[12] Jadhav, A. R., \& Rajalakshmi, P. (2020), Enhanced LoRa Data Rate through PATCH, In 2020 IEEE 6th World Forum on Internet of Things (WFIoT) (pp. 1-6). IEEE.

[13] Tsai, K. L., et al. (2020), Secure Session Key Generation Method for LoRaWAN Servers, IEEE Access, 8, 54631-54640.

[14] Xu, W., et al. (2019), Measurement, Characterization, and Modeling of LoRa Technology in Multifloor Buildings, IEEE Internet of Things Journal, 7(1), 298-310.

[15] Dobra, I. M., et al. (2020), LoRa propagation test in an urban environment and office buildings, In 2020 IEEE International Conference on Automation, Quality and Testing, Robotics (AQTR) (pp. 1-5). IEEE.

[16] Glória, A., et al. (2020), Water Management for Sustainable Irrigation Systems Using Internet-ofThings, Sensors, 20(5), 1402.

[17] Basford, P. J., et al. (2020), LoRaWAN for smart city IoT deployments: A long term evaluation, Sensors, 20(3), 648 .

[18] Kongsavat, A., \& Karupongsiri, C. (2020), Path Loss Model for Smart Meter on LoRaWAN Technology with Unidirectional Antenna in an Urban Area of Thailand, In 2020 IEEE International Conference on Computational Electromagnetics (ICCEM) (pp. 260262), IEEE. 
[19] Fernandez, L., et al. (2020), Assessing LoRa for Satellite-to-Earth Communications Considering the Impact of Ionospheric Scintillation, IEEE Access, 8, 165570-165582.

[20] Kouzinopoulos, C. S., et al. (2019), AMANDA: an autonomous self-powered miniaturized smart sensing embedded system, In 2019 IEEE 9th International Conference on Consumer Electronics (ICCE-Berlin) (pp. 324-329), IEEE.

[21] Mayer, P., et al. (2020), RTK-LoRa: HighPrecision, Long-Range and Energy-Efficient Localization for Mobile IoT devices, In 2020 IEEE Sensors Applications Symposium (SAS) (pp. 1-5), IEEE.

[22] SX1272 / Long Range, Low Power RF Transceiver 860-1000MHz / Semtech.

Available:

https://www.semtech.com/products/wireless-rf/loratransceivers/sx1272.

[23] SX1276 / 137MHz to 1020MHz Long Range Low Power Transceiver / Semtech.

Available:

https://www.semtech.com/products/wireless-rf/loratransceivers/sx 1276 .

[24] SX1261 / Long Range Low Power LoRaR RF Transceiver $+15 \mathrm{dBm} /$ Semtech.

Available:

https://www.semtech.com/products/wireless-rf/loratransceivers/sx1261.

[25] Souifi, J., et al. (2020), Smart Home Architecture based on LoRa Wireless Connectivity and LoRaWAN® Networking Protocol, In 020 1st International Conference on Communications, Control Systems and Signal Processing (CCSSP) (pp. 95-99). IEEE.

[26] Ferrari, P., et al. (2020), On the use of LoRaWAN for the Internet of Intelligent Vehicles in Smart City scenarios, In 2020 IEEE Sensors Applications Symposium (SAS) (pp. 1-6), IEEE.

[27] Ameloot, T., Van Torre, P., \& Rogier, H. (2020), LoRa Base-Station-to-Body Communication with SIMO Front-to-Back Diversity, IEEE Transactions on Antennas and Propagation.
[28] Ameloot, T., Van Torre, P., \& Rogier, H. (2020), Experimental Parameter Optimization for Adaptive LoRa Modulation in Body-Centric Applications, In 2020 14th European Conference on Antennas and Propagation (EuCAP) (pp. 1-5), IEEE.

[29] Lange, S., Pohl, J., \& Santarius, T. (2020), Digitalization and energy consumption. Does ICT reduce energy demand?, Ecological Economics, 176, 106760.

[30] Boquet, G., et al. (2020), LoRa-E: Overview and Performance Analysis, arXiv preprint arXiv:2010.00491.

[31] Paredes, M., Bertoldo, S., Carosso, L., Lucianaz, C., Marchetta, E., Allegretti, M., \& Savi, P. (2019), Propagation measurements for a LoRa network in an urban environment, Journal of Electromagnetic Waves and Applications, 33(15), 2022-2036.

[32] Bertoldo, S., Paredes, M., Carosso, L., Allegretti, M., \& Savi, P. (2019), Empirical indoor propagation models for LoRa radio link in an office environment, In 2019 13th European Conference on Antennas and Propagation (EuCAP) (pp. 1-5). IEEE.

[33] Sales Mendes, A., et al. (2020), Multi-agent approach using LoRaWAN devices: An airport case study. Electronics, 9(9), 1430.

[34] Kufakunesu, R., Hancke, G. P., \& Abu-Mahfouz, A. M. (2020), A survey on Adaptive Data Rate optimization in LoRaWAN: Recent solutions and major challenges, Sensors, 20(18), 5044.

[35] Singh, R. K., et al. (2020), Leveraging LoRaWAN Technology for Precision Agriculture in Greenhouses, Sensors, 20(7), 1827.

[36] Noura, H., Hatoum, T., Salman, O., Yaacoub, J. P., \& Chehab, A. (2020), LoRaWAN Security Survey: Issues, Threats and Possible Mitigation Techniques, Internet of Things, 100303.

[37] Cotrim, J. R., \& Kleinschmidt, J. H. (2020), LoRaWAN Mesh Networks: A Review and Classification of Multihop Communication, Sensors, 20(15), 4273. 